\title{
LA POESIA DI KAROL WOJTYŁA: UN ITINERARIO ASCETICO*
}

Nella raccolta delle poesie giovanili di Karol Wojtyła curata da Marta Burghardt esiste un gruppo di 17 sonetti, contenuti in una lettera del 14 novembre 1939 indirizzata dal giovane poeta all'amico professore Mieczyslaw, accompagnate peraltro da un commento dello stesso Wojtyła. L'interesse di tali testi, ormai a disposizione dei lettori, consiste nel fatto che si presentano a tutt'oggi, come vere primizie in nostro possesso. Il secondo sonetto in forma di lettera è particolarmente significativo in quanto contiene l'affettuosa richiesta all'amico di non disperdere queste poesie iniziali ma piuttosto di custodirle almeno nella memoria: «Rammenta il mio primo fascio di versi» (PG 41).

Nel commento che accompagna l'invio, interrogandosi sull'origine del suo poetare, egli aggiunge: «Eseguiti nel dolore e nello sgomento [...] Soltanto brandelli, pezzetti, primissimi inizi della sintesi, i più pallidi riflessi dell'albeggiare. Comunque l'albeggiare» (33). Dunque: l'albeggiare. Ma l'albeggiare di cosa? L'albeggiare della poesia? Della ricerca di senso? Del desiderio di dialogo? Dell'attesa di risposte e di segni? Del significato della vita? Della fede?

A ben leggere ci si accorge che tutte queste cose sono presenti nei «primissimi brandelli» dell'animo inquieto del giovane Wojtyła sulla soglia dei vent'anni, quando, appena uscito dal liceo, egli sta per aprirsi alla nuova scelta della vita universitaria.

Prof. Lia Fava GuZzetta - Università LUMSA (Roma); e-mail: liafava2@gmail.com

* Le citazioni delle poesie di Karol Wojtyła sono tratte dalle seguenti raccolte: Le poesie giovanili (cura e traduzione di Marta Burghardt), Studium, 2004 (citata nel testo con la sigla PG); Tutte le opere letterarie (presentazione di Giovanni Reale), Bompiani, 2001(citata nel testo con la sigla TOL); Giovanni Paolo II, Trittico romano. Meditazioni (a cura di Giovanni Reale), Bompiani, 2003 (citata nel testo con la sigla TR). 
Al fondo del suo animo, è comunque ancora viva la ferita profonda della morte della madre che ha segnato la sua vita di bambino: un vuoto, una nostalgia che richiede ancora di essere colmata e che si esprime come un'attrazione verso la parola poetica: «sulla tua tomba bianca / sbocciano i fiori bianchi della vita»: è poesia nell'elaborazione del ricordo dell' angoscia infantile che rende presente la mancanza di quell' "amore spento», la sensazione del tempo che è già passato, e la tristezza della continua inesauribile preghiera della «bocca esausta»: «Dona eterno riposo» (PG 41). Un enorme bisogno di riempire un abisso interiore, dunque, un vuoto di affetto!

La poesia nasce da una mancanza. Sempre il poeta colma con la poesia il vuoto di amore, dando con la concreta realtà della «creazione» poetica, la misura specialissima di una risposta alla sua sete. E così è anche per il giovane Lolek che, non a caso, in queste lettere, si firma con quel diminutivo che, probabilmente, nel ricordo ancora accarezza il suo orecchio col dolce suono della voce materna. La poesia nasce da una mancanza! Sì! E tutto evoca un'esperienza di mancanza in questi primi documenti della formazione interiore del giovanissimo poeta Karol. La situazione storica esterna è anch'essa continua fonte di sottrazioni, di negazioni, di tragiche privazioni a causa dell'invasione nazista della Polonia proprio nel '39: vanno tutte infatti dall'ottobre del ' 39 all'ottobre del ' 40 le lettere che accompagnano i versi. L'occupazione della patria è una frattura profonda, e non solo perché colpisce il cuore del paese amatissimo, ma anche e soprattutto per la conseguenza della separazione da tutto un mondo costituito dalla compagnia degli amici, e per l'allontanamento dell'esercizio di quella cultura identitaria nella quale era immersa la riflessione di quegli anni di formazione e di applicazione vitale di quella stessa cultura, insieme al gruppo dei compagni, studenti e attori, protagonisti di una forma di teatro sperimentale che, com'è noto, li aveva uniti appassionatamente. L'abbandono di tutto ciò e del luogo stesso, col trasferimento a Cracovia, dall'amata Wadowice natale, determinano questi «canti della primavera dell'anno tragico [...] eseguiti in mezzo a risonanze spaventose $[\ldots]$ eseguiti nel dolore e nello sgomento» com'è detto nella lettera di quel novembre 1939 definito «anno di guerra» (PG 33, 35).

Questi canti testimoniano lo sconvolgimento profondo dell'interiorità del poeta che si confessa accoratamente all'amico: «Da quel tempo ho attraversato la profondità della caduta, della disperazione e il burrone abissale della negazione» (PG 33). Queste parole, fanno luce sull'origine di una coscienza nuova che prende forma letteraria insieme all'inizio di una religiosità o di una tensione metafisica che andrà sempre più chiarendosi ma che qui è custodita come in un prezioso incunabolo.

In questo momento di speciale introspezione il giovane poeta ritrova nella sua memoria il permanere dei segni dalla sua origine e identità slava e a questa intimamente connessi i fondamenti della sua formazione culturale classica e umanistica, 
scoprendo progressivamente una visione della vicenda umana e storica che lo spinge ad elaborare una prima intima relazione col mondo e con la realtà intera. Di questo primo percorso interiore $\mathrm{i}$ testi giovanili recano profonda testimonianza. La fondamentale traccia che resterà presente in tutta la vita di Woityła è la coesistenza in lui di due dimensioni profonde della sua sensibilità e della sua cultura, la compresenza insopprimibile della sua «anima slava» e della sua classicità intellettuale, un innesto destinato a non offuscarsi mai e a rivelare sempre più quel binomio inscindibile che caratterizzerà il suo talento comunicativo ecumenico e la sua visione teologica della Chiesa. «Io sono greco e slavo» dirà di qui a poco in Convivio, un componimento da lui stesso definito «poema su se stesso» (PG 77-95). Qui, nelle prime prove, queste due dimensioni originarie si fondano nell'elaborazione sempre più evidente della metafora musicale che è prioritaria lungo tutto il gruppo delle poesie giovanili fino al Magnificat e che comunica al lettore quella concezione «armonica» dell'universo di origine tanto greca che cristiana rintracciabile nei filosofi classici (Pitagora, Platone etc.) e in Agostino, Ambrogio e nei Padri della Chiesa. Nella dolorosa elaborazione della nostalgia per la perdita della patria, nel pianto per l'abbandono, si innesta un'immersione nell'anima della Polonia, nella dimensione slava dell'intrinseco rapporto con la natura, gli alberi, i pioppi, le vette, tanto che il poeta tende l'orecchio a raccogliere - attraverso la «marcia del vento mattutino» - i suoni della «Polonaise», «l'arcobaleno delle melodie», mentre il vento stesso «suonerà l'organo boschivo» impersonando esso stesso l'organo. Le tante metafore musicali disseminate nei testi riveleranno sempre più l'anima slava del poeta e progressivamente esse testimonieranno il passaggio dal canto popolare della «polonaise» all'Inno vero e proprio:

- Polonaise passa sugli alberi ed io rimango tremante

e raccoglierò i suoni ultimi con i fiori su pascoli di montagna

- e sulle più taciturne fonti di quest'anima pregherò con un inno. (PG 47)

Il passaggio dai Sonetti all'Inno nell'impegno poetico, costituisce un accrescimento stilistico e contenutistico poiché segnala un ulteriore percorso interiore verso l'esprimersi di una religiosità consapevole e personale indicata anche nel lessico con l'apparire della parola «offerta». Significativo in tal senso il VI sonetto:

All'anima sia concesso passare dalla polonaise all'inno.

$[\ldots]$

Colpisci il mio cuore con l'inno, con un'offerta di mille incensi.

$[\ldots]$

Eccomi come santuario che spunta fra le nostalgie.

$[\ldots]$

Sono nell'offerta dell'organo (PG 49) 
A poco a poco la musica, il canto, l'organo diventano l'equivalente del poeta stesso che offre la sua voce: «E io sono per l'umanità il Miserere dei Salmi» (49), dirigendosi verso una esperienza di preghiera che prelude ad una vera via di contemplazione destinata a tracciare un lungo percorso.

È possibile affermare che questa disposizione all'offerta di sé che non è ancora la chiarezza della scelta sacerdotale ma che nasce dall'attenzione ansiosa alle drammatiche vicende storiche del momento e dall'immedesimazione nella tragica condizione di sofferenza del proprio popolo e dei propri compagni oppressi, crea in lui quell'inquietudine che secondo quanto ha affermato il mio maestro Giorgio Petrocchi, grande studioso di poesia mistica antica e moderna, «non trova giustificazione nel ragionamento della mente» ma piuttosto attinge «alcunché di misterioso che si affaccia alla sensibilità dell'uomo» e che è all'origine della poesia religiosa (Petrocchi 135). Ora questi testi poetici del primo Wojtyła testimoniano proprio l'approssimarsi di un senso del Mistero alla base della spinta verso la parola poetica e configurano la proposta di una visione musicale nella logica creativa di Dio così come è stata trasmessa dalla tradizione cristiana capace di incrociare e fare tesoro delle intuizioni già appartenenti al mondo del pensiero greco-romano. Nella riflessione del giovane Wojtyła infatti c'è come una sintesi meravigliosa tra l'idea dell'armonia del mondo (l'armonia delle sfere che risale a Pitagora), lo stupore del creato in accordo con il Creatore cantato nell'Inno di Ambrogio e caro ad Agostino, Paolo etc. e la visione di un universo creato come dono musicale da Dio stesso che «si china sull'arpa» (PG 145) per trasmettere all'uomo la sua musica e renderlo capace di canto:

- Oh Musica! Melodia! Musica!

Tu Onnipotente! Armonia della natura!

- Premi con il peso dell'arpa vibrante,

con le corde mi conduci ovunque sopra la terra,

fai risuonare con il liuto il canto dell'anima mia. (PG 101-103)

Potremmo dire che la musica è riconosciuta come la cifra di Dio, in questi versi giovanili, tanto che i momenti drammatici, gli eventi negativi della storia stessa che si allontana da Dio, non a caso vengono presentati come anti-musica. Nel poema Ballata delle arcate di Wavel che esibisce il sottotitolo di «poema sinfonico», il poeta attraverso un percorso drammaticamente visionario produce una tragica allegoria della storia umana come un tempestoso concerto in cui il simbolo più forte è costituito dal pianoforte di Chopin, che ad un certo punto della lotta tra le ragioni terrene della violenza bellica e la musica, dono armonioso dell'operare di Dio, subisce la ferita nel suo stesso corpo materiale attraverso l'immagine del «pianoforte insanguinato» (PG 193) del «sangue sparso sulla tastiera» (PG 191) mentre risuona alta l'invocazione dell'Inno: 
Padre! Dona vittoria alla Musica! ... Padre!

Infondi la Musica negli abissi!

Armonia dei mondi. (PG 195)

La forma dell'Inno si impone ulteriormente come potenziamento della lode religiosa e rafforzamento della presenza metaforica della musica, nel poemetto Mousike 2 («scritto probabilmente nei primi mesi della seconda guerra mondiale» secondo Marta Burghardt), (PG 203) che è l'ultimo testo della raccolta giovanile e che non a caso viene proposto dallo stesso poeta con il sottotitolo di Sinfonia, nella quale la compresenza di più temi - ripresi dalla drammatica storia della Polonia come simbolo di un mondo di guerre e di morte, pattern di lotta perenne tra il bene ed il male, vera e propria «tempesta» sulla terra, da sempre in lotta con la melodia del cielo - crea nel poema l'icona stessa della sinfonia per produrre quella che il poeta ha definito «visione dei tempi» (PG 235). Questa «visione dei tempi» che chiude la raccolta mette anche in primissimo piano la voce stessa del poeta che si fa protagonista di un'opera di mediazione tra il mondo e Dio, lanciando un accorato invito al popolo perché ascolti la divina musica. «Ponete sulle spalle della Musica le sorti del mondo» (PG 231). L'individuazione di questo spazio di mediazione insieme all'assunzione dell'Inno come forma di rapporto con Dio costituisce anche un nuovo momento, iniziale, di una consapevolezza che non è ancora chiarezza di una vocazione specifica ma è già motivazione di una disponibilità che diverrà sempre più generosa fino all'offerta totale che si andrà chiarendo successivamente ma che trova la sua nascita proprio nella primavera-estate del 1939, stagione importantissima all'origine di due testi come Parola Logos e Magnificat che custodiscono tale inizio. In essi si afferma esplicitamente un «io» poetante che assume la responsabilità di dichiarare in prima persona la propria scoperta della Parola:

Ascolto le confidenze serali del giardino,
con la vista dell'anima rifletto sulla Parola -
...
Ascolto le confidenze serali del giardino,
con la vista dell'anima Ti vedo - Parola. (PG 119, 121)

La Parola, divenendo oggetto di visione, diventa «Luce d'amore - trasparenza dorata / Parola si fece Carne - Compimento», mentre per colui che sta maturando una coscienza di sé, per l'artista,

Occorre le Parole prima abbracciare con una cinghia

per poi spingerle nel ritmo dell'Amore assoluto

- e creare un poema infiammato

$[\ldots]$

occorre trasformare in visioni lunari

le parole, che si intrecceranno nell'alloro dell'anima. (PG 127) 
È un'emozione per il lettore assistere al primo emergere di questo tema, di questa esperienza della «visione» («...hai fatto piovere nelle mie corde la Melodia / e in questa melodia Ti sei svelato in visione - attraverso il Cristo») (PG 145), che significa anche assistere al primo barlume della scoperta, da parte del giovanissimo Karol, del mistero dell'Incarnazione («Parola si fece carne»): il Mistero per eccellenza che costituirà l'oggetto di una sempre più profonda e intima contemplazione fino alla finale immedesimazione nella Croce e nella Passione di Cristo verso la quale tenderà già fin d'ora, come in abbozzo, la crescita ascetica di papa Wojtyła. Da questo momento inizia un tempo di formazione così prezioso che sarebbe da registrare tappa per tappa, da monitorare, come si dice oggi, in tutte le sfumature, e che, comunque, costituirà oggetto di studio per gli storici del futuro e soprattutto per gli studiosi di letteratura religiosa dell'era moderna che sicuramente potranno annoverare Wojtyła fra i poeti religiosi del secolo appena trascorso.

Anche se nelle poesie giovanili sono già rintracciabili dei semi riconducibili all'itinerario spirituale di Wojtyła, in particolare con l'intuizione del valore oblativo della parola già espresso in Parola - Logos («le parole di offerta richiedono un sacerdote [...] Dalla Parola lievita e costruisci un'offerta!») (PG 123, 133) non si può non segnalare la specifica singolarità del decennio che segue a questa prima basilare intuizione non ancora chiara come personale vocazione ma che andrà maturando via via, e che la biografia illumina perfettamente. Dopo il 1940 infatti le scelte di questo singolare giovane poeta si dirigono verso le due strade principali che caratterizzeranno tutta la sua vita: la comprensione della condizione umana e il desiderio di penetrare sempre più nella conoscenza dell'amore di Dio verso la creatura, attraverso la costante meditazione sul mistero dell'Incarnazione. Il Canto del Dio nascosto e il Canto del sole inesauribile del 1944, che si trovano nella raccolta delle opere letterarie edita da Bompiani nel 2001, possono apparire al lettore come una sorgente viva di indizi e segnali di una intima, ricca e complessa elaborazione interiore dell'esperienza mistica che comincia a profilarsi, e che porterà alla definitiva scelta sacerdotale attraverso dolorosi eventi di progressive perdite e faticose esperienze umane vissute nel quinquennio: l'inizio del suo lavoro nella cava di pietra nel '40, la morte del padre nel '41, l'uccisione da parte della Gestapo di un suo compagno che insieme a lui frequentava il seminario clandestino nello stesso periodo. Eventi fortemente incisivi interiorizzati attraverso la preghiera e la meditazione su questo tempo di passaggio dalla parola al discorso nel quale certamente prende sempre più forma quello che potremmo definire il suo «metodo», già così chiaramente espresso in una lettera proprio del' 40 all'amico Kotlarczyk, citata da Marta Burghardt: «Io con queste poesie sto appena imparando a parlare, prima di cominciare il discorso» (PG 267). 
Sono eventi che scavano un baratro di solitudine nell'animo di Karol sia nell'esperienza della lontananza fra gli uomini a causa della separazione sociale della povertà e del lavoro disumano cui molti sono costretti, sia a causa della morte che crea un incolmabile vuoto di presenze e strappa crudelmente gli affetti dal cuore. Dove cercare consolazione alla totale solitudine? Come collegarsi all'altro, alle sofferenze altrui? Cosa scoprire come possibilità di aiuto, di amore, di dono per vincere il dolore del mondo?

È possibile dire che questo tempo si configura per il poeta come una totale immersione nel silenzio, nella scoperta di un abisso, senza confini, senza contorni. Se è vero ciò che Giorgio Petrocchi ha colto nello studiare così attentamente le vite e gli itinerari di Mistici e Asceti, e cioè che «la maturazione del processo ascetico nasce per la più dal momento in cui si consuma l'esperienza giovanile, così in Francesco d'Assisi, in Jacopone, nel beato Giovanni Colombini, in un lento effettuarsi dei fenomeni di disgusto della vita del mondo» (Petrocchi 164), possiamo considerare segni di tale inizio anche $i$ testi poetici che abbiamo or ora indicato. L'immersione nel silenzio come necessità dello spirito attuale di Karol nasce proprio dal vuoto e dal disgusto che egli sente intorno a sé, avvertendo che proprio dalla penetrazione di quel silenzio può nascere l'acquisizione di una nuova profondità:

Lontane rive di silenzio cominciano appena di là dalla soglia .

Non le sorvolerai come un uccello .

Devi fermarti a guardare sempre più in profondità

finché non riuscirai a distogliere l'anima dal fondo. (TOL 45)

È chiaro che si tratta di un inizio irreversibile:

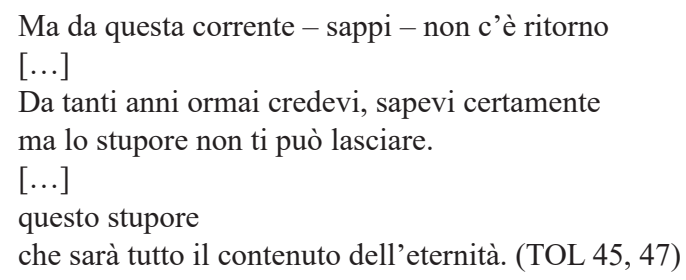

Lo stupore che avvolge da ora in poi l'interiorità del poeta sarà la via della comprensione di una nuova ricerca di reciprocità col divino attraverso il ridimensionamento della stessa via razionale e intellettiva alla base dell'inquieta riflessione sul senso della scienza e della ragione rispetto alla fede. C'è come un bilancio affidato ad una nuova coscienza di se stesso che si fa strada. Non si tratta più solo di conoscere, ma piuttosto di scoprire lo spessore autonomo della fede, esperienza intima di unione, non di ragionamento o di logica scientifica o di normale misura matematica. Non serve più il confronto con Pitagora, «eterno filosofo» (PG 231) che «misura, rimi- 
sura, misura» (PG 215), ora che il poeta s'inabissa, non solo con l'intelletto, nella ricerca del «Dio nascosto» per indirizzargli il suo «canto»:

Annegare, annegare!

[...]

solo l'anima, l'anima dell'uomo immersa in una minuscola goccia

l'anima rapita dalla corrente. (TOL 47)

Nascono nuove immagini, si creano nuovi simboli: il mare, la luce, per dar conto di questo esser preso, «avvolto»:

Quando il mare rapidamente ti nasconde

e ti scioglie in abissi silenziosi

- la luce strappa bagliori verticali alle onde languide

e il mare piano finisce, affluisce un chiarore

[...]

In quell'istante - guarda dentro di te. Ecco l'Amico

che è solo una scintilla, eppure è tutt'intera la Luce.

Accogliendo dentro di te quella scintilla

non scorgi altro,

e non senti di quale Amore sei avvolto.

L'amore mi ha spiegato ogni cosa. (TOL 47, 49)

Il procedere ascetico appare luminoso, e il suo divenire «fede e pienezza» si colloca pienamente nella consapevolezza del poeta:

Il Signore, quando attecchisce nell'intimo è come un fiore

assetato di caldo sole.

Vieni, dunque, o luce, dalle profondità dell'inesplicabile giorno,

e posati sulla mia riva. (TOL 51)

Così implora il poeta mentre comprende che per giungere alla luce dovrà prima inabissarsi nell'oscurità, perdersi nel buio dell'incomprensione: «Più aguzzo lo sguardo, meno riesco a vedere» (TOL 53), per potere elaborare quasi un sistema di attesa per una nuova rivelazione che può avvenire soltanto in uno spazio totale, in uno svuotamento di sé, in un nulla che attenda una diversa pienezza. È così che è possibile che si maturi l'esperienza mistica che potrà condurre alla scelta del dono di sé, con la rinunzia perfino al ruolo dell' intelletto o al gusto della propria parola:

Io stacco piano la luce dalle parole

e raduno i pensieri come un gregge di ombre

e lentamente in tutto immetto il nulla

che attende l'alba della creazione. 
Lo faccio per creare uno spazio

alle tue mani tese

lo faccio per avvicinare

l'eternità in cui Tu possa alitare...

Inappagato dall'unico giorno della creazione

io bramo un nulla crescente,

perché il mio cuore sia disposto al soffio

del Tuo Amore

Per quest'attimo - colmo di strana morte

che salpa verso l'eterno infinito,

e per un tocco di lontana arsura

che fa languire il profondo giardino.

Si confondono l'attimo e l'eterno

la goccia ha risucchiato il mare -

e un solare silenzio

cala sul fondo dell'estuario.

La vita è forse un'onda di stupore, un'onda più alta della morte? (TOL 53-55)

Come si vede c'è come un racconto della propria esperienza interiore, quasi lo svelamento di una autopedagogia spirituale, che lascia interessanti tracce del percorso agli inizi, tendente a liberarsi del già acquisito mentalmente per conquistare intero lo spazio della vera fede: «Dunque, sapere sempre di meno e credere sempre di più ... Penso spesso a quel giorno di visione» (TOL 59-61), mentre la tensione contemplativa tende a farsi sempre più preghiera:

Ti ringrazio perché hai posta la dimora dell'anima lontano d' ogni fragore

$[\ldots]$

Poiché tu sei il silenzio stesso, questo grande Tacere,

da ogni suono di voce fammi libero,

ed entra in me Tu solo, col Tuo fremente essere. (TOL 61)

E dalla preghiera nasce l'interlocuzione, il dialogo, la scoperta di un originario atto di predilezione e di scelta:

Quando dall'infinito facesti emergere il tempo

per appoggiarlo all'altra riva,

Tu già sentivi il mio lontano pianto, ne sapevi da secoli il motivo. (TOL 65)

Si tratta di una scoperta che agisce come un fulmine che accende il fuoco del desiderio nell'animo turbato ed incantato scatenando una incommensurabile nostalgia 
Tornare sempre a quel tempo quando cullato solo dal Tuo Pensiero

ebbi in me più innocenza di un bambino, e al preconfezionato

e più profonda trasparenza. (TOL 69-71)

È possibile dire forse, a questo punto, che proprio su questa nostalgia di originaria trasparenza si realizzi l'approdo del giovane poeta alla mistica sponda dell'offerta della sua vita. La stessa sua biografia consente di conferire a questo periodo un particolare rilievo in tal senso, soprattutto se ricordiamo che sono questi gli anni della scoperta e della appassionata lettura dell'Opera di San Giovanni della Croce assorbita fino al punto da far nascere nel giovane Karol il desiderio di entrare nel Carmelo. Sappiamo che ciò non è poi avvenuto (era un'altra la vocazione provvidenziale!) ma non è certo un caso che, fra qualche anno, nel 1948, Wojtyła, già sacerdote, sceglierà per la propria tesi di dottorato un argomento che riguarderà «La dottrina della fede in S.Giovanni della Croce» (Wojtyła, La dottrina della fede). Sono tanti, nei testi poetici che stiamo analizzando, gli spunti che hanno la loro originaria ispirazione in questa preziosa fonte, come ha messo bene in evidenza, Santino Spartà (Spartà, L'opera poetica completa). Tutta la storia mistica, se così possiamo dire, di San Giovanni Paolo II, dimostra la permanenza di tale modello fino alla fine. Qui, nella sezione intitolata Il canto del sole inesauribile c'è evidente come un'ansia dell'anima che si dibatte fra timore e tremore, luce e ombra, sole pieno e crepuscolo, alba e tramonto:

Il Tuo sguardo fisso sull'anima, come il sole verso la foglia s'inclina, ne arricchisce il fiorire con la profonda, trasparente bontà,

l'accoglie nel suo raggio

- ma tu, Maestro, guarda:

che accadrà della foglia e del sole? - la sera si avvicina. (TOL 63)

Il poeta si interroga attraverso l'immagine della foglia e del sole, sul rapporto tra la piccola anima e l'immensità divina attraverso situazioni evocative di grande suggestione che mostrano come Dio sappia intervenire rivelandosi in modo speciale attraverso l'iniziale apparire del Mistero:

Così, per non lasciarmi solo

in quell'ansia, spogliasti

il crepuscolo d'ogni suo orrore

e all'eternità desti il sapore del pane. (TOL 65)

È visibile qui l'intuizione eucaristica e l'itinerario di comprensione e progressivo disvelarsi della paternità di Dio che l'anima va conquistando attraverso la rilettura della narrazione evangelica e il ritrovarne nella memoria interiore i protagonisti: Maria, Nicodemo, la Samaritana, tutti segni disseminati in quella storia donata all'uomo 
dalla venuta di Cristo nel mondo, dalla sua parola, dal suo sacrificio sulla croce e dall'evento del dono eucaristico. Tutto è contenuto in un'unico nodo che si offre alla rivelazione mistica:

Mi abbasserò, fratello

mi abbasserò, non lascerò mai soli i tuoi occhi,

e mi nasconderò dapprima nella croce,

poi, come il pane, nel grano maturo. (TOL 67)

È come sentire la voce diretta di Dio, che invita alla meditazione contemplativa, all' introspezione, alla progressiva accoglienza, quando ancora il giovane è «come un torrente» che corre «troppo in fretta» non ancora degno di «quel fondo così abissale», che il Signore visita ogni giorno («Là, ogni giorno, il mio Signore viene e resta») (TOL 73) donando la sua infinita presenza al «fallibile pensiero» del piccolo poeta che, persistendo il suo accorato senso di impotenza, vorrebbe restare nel silenzio del godimento mistico:

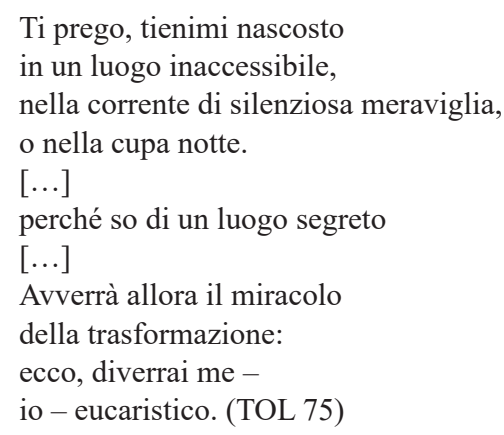

C'è quasi un desiderio simile a quello di quegli apostoli che chiedevano a Gesù di restare con loro al farsi della sera. Ma, all'apice di questo totale desiderio di possesso e di immedesimazione due testi emblematici del 1950 chiariscono l'intensità dell'impegno preliminare di ascolto delle scritture per potere meglio vedere e rispondere alla chiamata. Il primo, Canto dello splendore dell'acqua, si concentra interamente sulla rievocazione dell'episodio della Samaritana e poeticamente si costruisce sul simbolo dell'acqua, così presente e così vicina, tanto che la puoi sentire, toccare, il suo luccichìo ti attira e ti affascina:

Guarda - senza posa si sfalda in scaglie d'argento -

e trema in essa il peso della profondità

$[\ldots]$

L'acqua lava dai tuoi occhi i cerchi di stanchezza

e ti lambisce il volto con riflessi di larghe foglie.

Tanto lontana la sorgente - 
$[\ldots]$

il pozzo crea nel tuo sguardo solo barlumi di foglie,

con chiazze di verdi riflessi vela dolcemente il tuo volto

laggiù - sul fondo.

Quanto è lontana ancora la sorgente? (TOL 125)

Sorge la improcrastinabile e pressante interrogazione. Col proiettare sulla Samaritana le inquietudini e le domande («dov'è la sorgente?») il poema si drammatizza, il pozzo appare sempre più in mezzo alla scena e acquista il ruolo simbolico di specchio nella cui profondità si custodisce il passato e il presente della donna («di me com'ero prima e poi») mentre l'immagine simbolica accresce la sua valenza significante concentrando su di sè l'esperienza di una «meditazione rinnovata», come viene detto, presentandosi come «varco» che consente di arrivare al fondo, di vedere: infatti «il pozzo mi ha introdotta nei suoi occhi, / in essi mi ha chiusa» (TOL 133). Proprio così facendo viene costruito un «varco» verso l'unione, «un semplice varco nel muro che finora attraversavo» (TOL 133), verso l'unione, espressa attraverso le parole della Samaritana:

Il pozzo a Te mi ha unita il pozzo in Te mi ha fatta entrare.

Altro non v'era, tra noi, che il suo splendore profondo,

- vibrante come chiara pupilla nell'orbita di pietra. (TOL 133)

Come si vede il linguaggio è molto analogico e forte, e questa chiara pupilla che vibra nell'orbita di pietra, crea una sinestesia di rara suggestione derivante dal contrasto tra il vibrare dello splendore della chiara pupilla e la statica fissità dell'orbita pietrificata, che indica proprio il distacco dalla precedente condizione e l'avvento della metamorfosi interiore. La rievocazione del vangelo, dunque, il riapparire delle figure emblematiche di quella narrazione che deve diventare annunzio per gli apostoli, per i cristiani e per la Chiesa, è d'ora in poi la via da seguire per rintracciare i modelli e unirsi a loro. Il modello per eccellenza, a questo punto, per il neo-sacerdote Wojtyła, sarà Maria, la madre, le cui caratteristiche, che scaturiscono da una immedesimazione meditativa di eccezionale penetrazione umana e psicologica, disegnano via via la fisionomia della Chiesa nella sua funzione di materna protettrice dell'intera umanità. Nel poemetto del 1950, La Madre, poeticamente, attraverso l'intima rivisitazione della vicenda umana della maternità eccezionale della Madonna, viene offerto al lettore non soltanto il segreto e misterioso legame dell'intimità mariana di Wojtyła, ma anche la sua visione ecclesiologica e teologica nella quale risulterà potenziata l'immagine della Chiesa/Mondo che assume le caratteristiche di Maria nel materno legame col figlio. Come in una lauda medievale si svolge l'intenso dialogo tra Maria, Gesù figlio mediatore con la storia e Giovanni emblema dell'allargarsi dell'amore 
oltre il figlio verso l'alterità degli altri uomini. Tutto si svolge attraverso la memoria di Maria, che rievoca con indicibile suggestione per il lettore, i momenti della sua vita di madre che a poco a poco entra nel mistero della vita e della missione di Gesù che lei sola segretamente intuisce conservandola segretamente nella sua gioia e nella sua sofferenza. Dalla tenerissima rievocazione del primo sussurro del bambino

Il mio spazio scorre nella memoria. Non svanisce

il silenzio di viuzze lontane, fermo nell'aria come vetro

che nelle pure iridi si sbriciola in luce e zaffiro -

Vicinissime le parole del bambino dalle quali il silenzio prende il volo

$<$ Mamma, Mamma $>$ (TOL 137)

fino al canto finale della totale comprensione e immedesimazione:

Mi avvolgerà il tempo nuovo,

$[\ldots]$

e tutto infine sarà colmo $[\ldots]$

Aprirò allora il mio canto, ne capirò ogni sillaba,

aprirò allora il mio canto, ch'è intento alla tua vita,

tutto pervaso dall'Evento, immensamente chiaro e semplice

che in ogni uomo germoglia, così palese e segreto

e che in me si è incarnato e rivelato

giungendo a molte genti, tra cui ha trovato dimora. (TOL 147)

Come ha detto bene Antonio Spadaro «il senso della contemplazione è nel lasciarsi immergere all'interno del mistero che si contempla» (Spadaro 42) ed a me pare si possa affermare che questo poemetto testimoni proprio un momento contemplativo di estrema immersione da parte di Wojtyła nel mistero di Dio-Cristo e nel mistero personale della sua chiamata e della sua visione ecclesiologica, perché sembra infatti che questi testi conservino il segreto della sintesi di un pensiero e di un'offerta, di una scoperta di sé e di una svolta di vita contenente una risposta aperta e definitiva. D'altronde intorno agli anni cinquanta, come la biografia ci informa, si coagulano i diversi livelli di creatività che costituiscono la personalità di un così speciale protagonista della ricerca di verità del ventesimo secolo: Karol Wojtyła. D'ora in poi all'immersione contemplativa si aggiungerà un'altra immersione, quella dentro il mistero della storia umana e dell'uomo in essa, anche sulla spinta dei compiti sacerdotali, ed ecclesiali, ma soprattutto come proseguimento dell'indagine su Dio, che ha abbracciato l'umanità col farsi egli stesso uomo, rivelando in tal modo quella parte del mistero concernente l'uomo in quanto tale, grande quanto quello della stessa esistenza di Dio: Dio/Uomo. Un bel problema per il filosofo e per il teologo, da esplorare con un'attenzione spasmodica e instancabile per capire l'uomo in sé e Dio in esso: l'Incarnazione: un orizzonte enorme, il Mistero più grande del quale cogliere 
tutti i segni possibili: da indicare all'uomo, da spiegare all'umanità sofferente, da fare accettare come atto d'amore da parte del creatore. È questa, dopo gli anni cinquanta la costante prospettiva di ricerca, di domanda e di risposta di Papa Wojtyła, il quale, sotto qualunque veste - parroco, vescovo, papa, poeta, scrittore, filosofo, creatore di Encicliche, viaggiatore nel mondo come nunzio del Vangelo - vuole aiutare i suoi contemporanei a superare la sofferenza dei limiti della storia offrendo loro la possibilità di una vera, liberante visione. Nel poemetto del ' 52 Pensiero - Strano spazio, nel quale i versi lunghi spesso fanno pensare a una meditazione in forma di prosa poetica, egli afferma con chiarezza: «credo che l'uomo soffra soprattutto per mancanza di visione [...] deve allora aprirsi la strada fra i segni» (TOL 117). Ed una lunga strada fra i segni è quella che da ora in poi il poeta esplorerà consegnando al lettore delle vere icone di tale esplorazione, nell'umano e nel divino, nel rapporto uomo/Dio, assecondando la sua vocazione poetica. E qui devo finalmente confessare il mio enorme rammarico di non conoscere la lingua polacca ritrovandomi purtroppo impedita dal gustare il vero linguaggio poetico di così grande autore, accontentandomi di leggerlo in traduzione e quindi di coglierne sopratutto lo spessore semantico e non la dimensione squisitamente poetica, musicale, metrica, fonosimbolica, che come sappiamo costituisce l'autentica fisionomia della poesia. Malgrado ciò, è innegabile che, come afferma Antonio Spadaro, «l'universo di immagini e di pensieri» che caratterizza la sua poesia, sopravvive «anche nel processo di traduzione» (Spadaro 13). La produzione poetica dei prossimi anni infatti, che possiamo considerare in un arco unico fino alle soglie del pontificato, può trovare una espressione globale in un significativo titolo ispirato dall'episodio della Veronica: La Redenzione cerca la tua forma per entrare nell 'inquietudine di ogni uomo che mostra lo sguardo di Wojtyła incessantemente attento ad ogni possibile indicatore di questa «forma»:

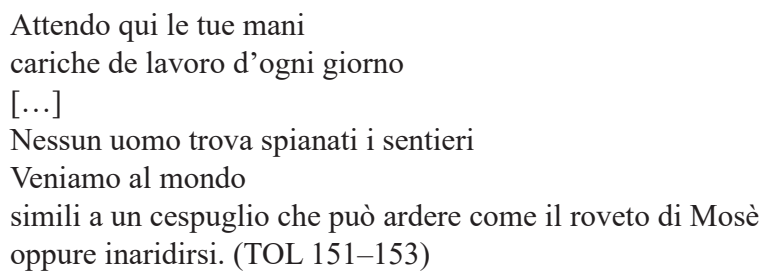

Si tratta, per il poeta, al momento, di esplorare la fisionomia dell'uomo, nella sua condizione esistenziale, e al contempo di fare emergere le tracce di un interesse dell'amore divino nei suoi confronti. Ne nasce una galleria di ritratti umani, che, attraverso la formula «profili di Cireneo», vengono convocati come icone dell'umanità esposta alla propria sofferenza ma anche all'amorevole iniziativa di Dio. Sono ritratti dell'uomo comune, «singoli profili»: Il melanconico, Schizoide, I ciechi, Attore, 
Ragazza delusa in amore, Fanciulli, Pensieri di un uomo, Descrizione dell'uomo, Operaio in una fabbrica automobilistica, Operaio in una fabbrica d'armi, Maddalena, Un emotivo, Uomo d'intelletto, Uomo di volontà, Simone di Cirene. Tutti ritratti dell'uomo, che evocano la vita stessa: il lavoro, l'amore, la sofferenza, e l'umanità sovente smarrita in essa, piena di ansie, spesso con domande senza risposta, senza un approdo. Dallo scavo dentro la condizione umana di sempre l'attenzione si sposta via via verso la contemporaneità, portando alla ribalta problematiche molto attuali nel passaggio dal secondo al terzo millennio con la voce di chi come lui le ha realmente vissute e può dare alla stessa parola poetica il valore di una testimonianza.

Nel suggestivo testo dal titolo La cava di pietra (1956) il poeta mette in scena il drammatico rapporto tra la fatica del lavoro materiale, la lotta contro la materia, e la rabbia interiore alla quale viene costantemente provocato l'uomo / operaio che soffre un tale peso. A conclusione del componimento, la sezione In memoria di un compagno di lavoro, vittima di un incidente, esprime una pietas struggente che evoca un canto funebre:

\section{1.}

Non era solo. I suoi muscoli si diramavano in una folla immensa

finché alzavano il martello, finché vibravano di energia -

ma questo durò solo finché egli sentì il terreno sotto i piedi,

finché la pietra non gli squarciò la tempia

e non gli entrò nelle stanze del cuore.

2.

Sollevarono il corpo: Sfilarono in silenzio:

3.

Da lui ancora emanava fatica e un senso d'ingiustizia:

Avevano bluse grigie, scarpe infangate fin sopra la caviglia:

$\mathrm{E}$ in quel modo rivelavano

che cosa tra la gente dovrebbe aver fine.

4.

Il suo tempo si fermò con violenza. Sui quadranti di bassa tensione

le lancette, liberate di colpo, scesero a zero.

La pietra bianca entrò in lui, corrose la sua essenza

e a sé l'assimilò tanto da farne pietra.

5.

Chi alzerà quella lastra? Chi sdipanerà di nuovo i pensieri

in quelle tempie squarciate - come si squarcia l'intonaco di un muro?

Lo stesero supino su un lenzuolo di ghiaia:

Venne la moglie disfatta.Tornò il bambino da scuola.

6.

Tutto qui? La sua rabbia sola dovrà passare negli altri?

Non maturava forse in lui con verità e amore?

Generazioni future devono forse sfruttarlo come grezza materia;

Privandolo della sua essenza più intima e unica? 


\section{7.}

Le pietre di nuovo si muovono - Il carrello sparisce fra i fiori.

Di nuovo una scarica elettrica incide la cava.

Ma l'uomo ha portato con sé la segreta struttura del mondo

dove l'amore prorompe più alto se più lo impregna la rabbia. (TOL 201-203)

A questa scena che ha quasi il sapore di una sacra rappresentazione, il poeta affida, iconicamente, tutto il senso di una riflessione sull'umano che concentra in un unico grumo doloroso la considerazione della sopportazione della fatica, la rabbia per l'ingiustizia, l'angoscia della perdita. La fatica del lavoro suscita emozione e commozione nell'animo del poeta che intensificherà con immagini nuove la dimensione metaforica del suo linguaggio attraverso il potenziamento dell'orizzonte allegorico della fragilità umana e della sua lotta per vivere :

[...] Prima nasce la fatica

come quando apri la porta contro la bufera,

quando ormeggi la barca controcorrente ad un esile palo. (85)

(la porta contro la bufera, la barca controcorrente, l'esile palo per l'ormeggio). La ricerca dei segni della fisionomia dell'uomo si allargherà dall'individuo singolo alla massa, parola tanto presente nell'epoca moderna, per individuare nella gente tutta nella «gente assorta», ad es. in attesa della cresima (TOL 81), nella gente chiamata comunemente «folla» - lo spazio antropologico nel quale approdano i confini di Dio: «E accanto scorre la folla, / donne, bambini, soldati: tutti si aggirano ai confini di Dio». Si allarga l'orizzonte della contemplazione, per il sacerdote, per il vescovo in una commistione di responsabilità e di ruoli che ne acuiscono la sensibilità, fino al punto da consentirgli di leggere tutto ciò che è «narrato» nel viso dell'uomo. Infatti:

Ciò che sgorga è congiunto con l'occulto, passa intatto il confine e nessuno, nessuno lo sfiora con lo sguardo perché i pensieri si affollano agli occhi come gli insetti sul vetro e quieti brillano nelle pupille ed in essi si addensano gli atti. (TOL 83)

Una visione integrale dell'uomo, dentro l'attenzione di Wojtyła che conosce le conquiste della modernità scientifica, che tiene presenti i tanti piani e le stratificazioni che la moderna biologia segnala in relazione alla complessità dell'essere umano. Il poeta/interprete inventa le figure che nel linguaggio della poesia possano esprimere tale complessità impressa dal Creatore, nell'inscindibile unione di corpo e spirito: «Lo spirito non vedrai mai / solo gli occhi sono specchio al pensiero» (TOL 83) e nel passaggio dal corpo (biologia, fisiologia) allo spirito (essenza interiore misteriosamente invisibile) coglie un intreccio delicato e fragile: il limite della corporeità e una inestinguibile sete di fronte al mistero: 
Nella strada i passanti dai corti giubbotti e dai capelli spioventi sul collo tagliano con la lama del passo

lo spazio del grande mistero

che in ognuno di loro si estende tra morte e speranza. (TOL 101)

La lama del passo che taglia il mistero del percorso umano concentra l'attenzione del lettore, con un'immagine suggestiva e fortemente concreta dando rilievo all'oggetto privilegiato dell'amorevole pensiero e dell'attenzione pastorale di Wojtyła: l'uomo in cammino, e il suo instancabile cercare.

Se cerchi il luogo dove si dibatteva Giacobbe,

non vagare fino ai paesi d'Arabia, non cercare sulle mappe il torrente, troverai molto più vicine le orme.

Lascia solo che nella prospettiva dei tuoi pensieri appaiano le luci degli oggetti. (TOL 121)

Appare chiaro il lessico della ricerca dei segni: vanno rintracciate «le orme» del passaggio dell'uomo sulla terra alla ricerca di un rapporto con Dio mai concluso e misterioso come una iscrizione che attende ancora di essere compresa: «Mistero un'iscrizione profonda / ancora non del tutto decifrata». Scoprire questo rapporto e volerlo indagare implica la volontà della fede e la scelta della testimonianza, e perfino del martirio. È ciò che ha caratterizzato il pontificato di Giovanni Paolo II, nell'ultimo tratto della sua ascesi. Forse non è un caso che l'ultimo testo poetico prima del pontificato abbia consegnato al lettore la splendida icona del martire Stanislao come un exemplum da meditare, vera «iscrizione» da decifrare:

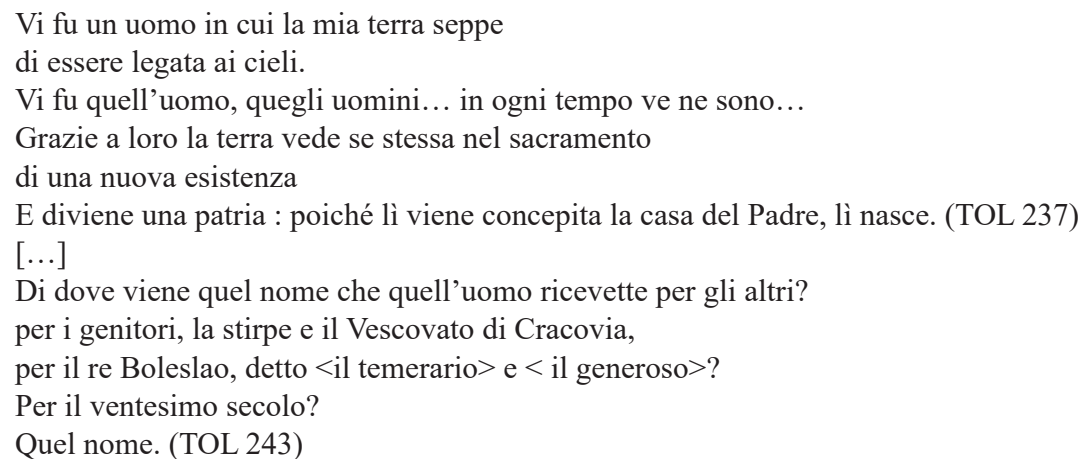

È chiara l'identificazione della responsabilità e dell'offerta nell'unione di fede, ed è chiara la scelta del modello del martire Stanislao che si può intravvedere come in un palinsesto, nell'ultima parte della vita di Wojtyła.

La finale meditazione poetica che egli ci ha lasciato appartiene a questo ultimissimo periodo e si rivela al mondo con la chiarezza dell'indicazione del nome del suo autore: Giovanni Paolo II, che non era mai apparso nelle precedenti pubblicazioni, 
affidate a pseudonimi. Quest'ultima opera è il Trittico Romano. In essa Wojtyła individua l'uomo come «lettore di frammenti» e se stesso come tale, di fronte alla grande «iscrizione» da decifrare rappresentata dalla michelangiolesca Cappella Sistina. Viene in mente Dante Alighieri:

nel suo profondo vidi che s'interna,

legato con amore in un volume,

ciò che per l'universo si squaderna. (Divina Commedia. Paradiso XXXIII, vv. 85-87)

Nella policromia che Michelangelo ha lasciato sulle mura della Sistina si apre realmente il Mistero:

$$
\begin{aligned}
& \text { Mi trovo all'ingresso della Sistina - } \\
& \text { Forse tutto ciò era più facile esprimere } \\
& \text { nel linguaggio della «Genesi»- } \\
& \text { Ma il libro aspetta l'immagine - È giusto } \\
& \text { Aspettava il suo Michelangelo. } \\
& \text { Perchè Colui che creava, «vedeva» - vide } \\
& \text { che «ciò era buono". } \\
& \text { „Vedeva", il libro perciò aspettava } \\
& \text { il frutto della «visione». (TR 43) }
\end{aligned}
$$

Non c'è quindi solo il racconto della creazione ma c'è la «visione», la pura, perfetta visione dell'atto creativo. Ed è qui, secondo Wojtyła, «la chiave» per leggere in profondità, la stupefacente «policromia», con la quale ci parla l'affresco michelangiolesco.

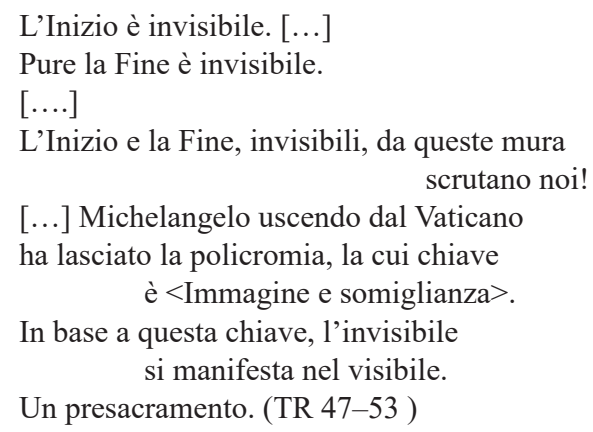

A questo punto è come se Wojtyła ci parlasse del suo stesso personale approdo alla fine del suo viaggio mistico. Egli è giunto alla «Visione» pura del Mistero, ma lo fa offrendo a noi una via tangibile di interpretazione, una «chiave» com'egli la chiama, rappresentata dalla policromia stessa del pittore, che offre allo sguardo l'uomo come Dio l'ha voluto: «immagine e somiglianza». L'opera del pittore con l'abbondanza, 
la magnificenza dei suoi colori, diviene essa stessa figura della molteplicità ed esuberanza della creazione divina.

L'ultima opera poetica di questo grande santo propone dunque, ancora la relazione visibile /invisibile e ci parla di una «rivelazione» riguardo a un si grande mistero del quale cercare i segni proprio nel viaggio dell'uomo sulla terra: la parte conclusiva del Trittico, infatti, è dedicata a questo viaggio.

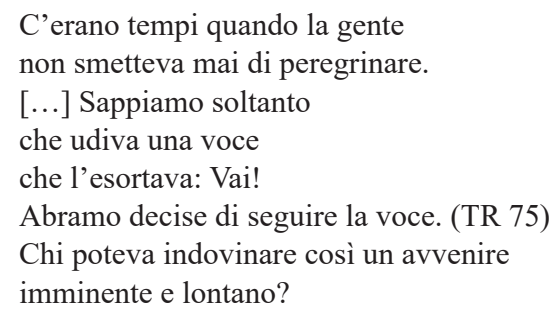

Chi è Costui, il Senza-Nome, che ha voluto rivelarsi nella voce?

Che ha parlato ad Abramo così come parla un uomo ad un altro uomo?

Era l'Insolito, Diverso da tutto ciò che l'uomo di Lui poteva pensare.

Parlava - quindi aspettava un risposta... (TR 79)

Ecco che alla fine del mistico viaggio Wojtyła ci presenta l'immagine di Dio nella sua assoluta «novità»: egli è l' «Insolito», il «Diverso». Chi gli risponde deve essere disponibile a questa imprevedibilità del nuovo. È possibile che l'uomo del ventesimo secolo, sempre più omologato e tecnologico, tendente alla ripetizione e al preconfezionato piuttosto che all'originalità, l'uomo robot del terzo millennio, al quale si rivolge Wojtyła, faccia fatica ad accettare il rischio della sorprendente promessa di Dio «farò nuove tutte le cose». Forse all'uomo di oggi sarà più difficile dare la risposta che Abramo ha dato. Ma questo grande poeta religioso si è congedato dal mondo indicandogli non soltanto le due grandi icone di Abramo e Michelangelo l'Inizio e la Fine del progetto di Dio per l'umanità, ma anche l'immagine della sua personale malattia e indicibile sofferenza - la sua Via Crucis - l'offerta del proprio corpo dolorante, per aiutare l'uomo contemporaneo a dare quella risposta.

Il suo «non abbiate paura» risuona ancora, per sempre, nella nostra memoria. Ed è uno splendido, poetico verso. Per la Speranza. 


\section{BIBLIOGRAFIA}

Dante Alighieri. Divina Commedia. Paradiso XXXIII, vv. 85-87.

Giovanni Paolo II. Trittico romano. Meditazioni, a cura di Giovanni Reale, Bompiani, 2003.

Petrocchi, Giorgio. „La religiosità”. Letteratura italiana, vol. V: Le Questioni, Giulio Einaudi Editore, 1986, p.135.

Spadaro, Antonio. Nella melodia della terra. JacaBook, 2007.

Spartà, Santino. L'opera poetica completa di Karol Wojtyła. Libreria Editrice Vaticana, 2013.

Wojtyla, Karol. La dottrina della fede in San Giovanni della Croce. Bompiani Editore, 2003.

Wojtyła, Karol. Le poesie giovanili, cura e traduzione di Marta Burghardt, Edizioni Studium, 2004.

Wojtyła, Karol. Tutte le opere letterarie. Presentazione di Giovanni Reale, Bompiani, 2001.

\section{LA POESIA DI KAROL WOJTYŁA: UN ITINERARIO ASCETICO}

\section{Sommario}

L'itinerario poetico di Karol Wojtyła viene esplorato nel suo evolversi a partire dalle Poesie Giovanili che privilegiano inizialmente la forma del Sonetto per passare all'Inno e alla Ballata. Successivamente vengono sperimentate forme più complesse ed aperte che riescono a fondere e superare il confine poesia / prosa in linea con le poetiche della modernità letteraria tra XX e XXI secolo. L'evoluzione del linguaggio poetico si svolge parallelamente all'approfondirsi della vicenda personale ed interiore del poeta sulla via della progressiva crescita di una esperienza mistica singolare ed insolita. Egli, per la sua capacità di immergersi ed immedesimarsi sia nella drammaticità storica del suo e del nostro tempo, sia nella ricerca inesausta di una verità che vada oltre «il magro fiume della storia» per rispondere alla sete di Assoluto dell'uomo, percorre una scala di Ascesi sempre più intensa le cui tappe in progress lasciano preziose tracce nei testi poetici, i quali acquisiscono via via una dimensione di iconicità sempre più significativa. Nell'ultima opera Trittico Romano (l'unica a firma Giovanni Paolo II) il poeta, sacerdote e mistico, giunge all'approdo della pura «visione» del progetto divino iscritto nella "policromia» stessa dell'affresco michelangiolesco della Cappella Sistina, assumendolo come vera, immensa Icona di «Immagine e Somiglianza», consegnata agli uomini dal pittore, messaggio e contemporaneamente «chiave di lettura», attraverso cui l'Invisibile si manifesta nel Visibile. Senza dubbio possiamo considerare Karol Wojtyła uno dei più grandi protagonisti della poesia religiosa fra i due secoli.

Parole chiave: Karol Wojtyła; Giovanni Paolo II; produzione letteraria; poesie; critica ed interpretazione

\section{POEZJA KAROLA WOJTYŁY: WĘDRÓWKA ASCETYCZNA}

$$
\text { Streszczenie }
$$

Artykuł jest swego rodzaju wędrówką po twórczości poetyckiej Karola Wojtyły - Jana Pawła II, wędrówką ascetyczną, a jednocześnie niezmiernie bogatą, w ślad za osobą z zewnątrz, wolną od naszego rodzimego kontekstu historyczno-kulturowego, zatem i otwartą na nieograniczone perspektywy interpretacji. Praca ukazuje uniwersalny charakter dorobku poetyckiego Karola Wojtyły, kładzie akcent na nowe elementy percepcji tejże poezji i w innym świetle ukazuje znaczenia wersów, wciąż tak bardzo aktualnych i niezmiennie adresowanych do człowieka, który także (a może zwłaszcza) dziś boryka się z odwiecznymi dylematami. Już we wczesnych utworach literackich obecne są (i na 
trwale pozostaną) dwa głębokie wymiary: dusza słowiańska oraz intelektualna klasyczność, które staną się charakterystyczne również dla talentu komunikacji ekumenicznej i wizji teologicznej Kościoła u Jana Pawła II. W przejściu od młodzieńczych sonetów do hymnów można dostrzec nie tylko rozwój stylistyczny i głębię przesłania, ale przede wszystkim podążanie w kierunku świadomej religijności, kontemplacji, w kierunku kapłaństwa, które rodziło się w szczególnie dramatycznych czasach drugiej wojny światowej. Z kolei w okresie kapłańskim wybory poety naznaczone są pragnieniem zrozumienia kondycji człowieka oraz zauważanie miłości Boga w stosunku do stworzenia. Kontemplacja staje się coraz bardziej modlitwą, a z modlitwy rodzi się dialog. Twórczość literacka Karola Wojtyły jawi się jako opowieść o własnym doświadczeniu wewnętrznym oraz odsłania duchową antropologię.

Słowa kluczowe: Karol Wojtyła; Jan Paweł II; twórczość literacka; poezje; krytyka i interpretacja.

\section{KAROL WOJTYŁA'S POETRY: AN ASCETIC JOURNEY}

\section{Sum mary}

This article is a kind of journey through the poetic work of Karol Wojtyła / John Paul II - a wandering that is ascetic, yet extremely rich - in the footsteps of an outsider, a person free from our native historical and cultural context, and hence open to the unlimited possibilities of interpretation. This work shows the universal character of Karol Wojtyła's poetic output; it emphasises new elements in the perception of that poetry, while showing the meaning of the verses in a different light, verses still relevant and invariably addressed to man, who is also (and maybe especially) struggling with eternal dilemmas today. The early literary works already contained two profound dimensions: the Slavic soul and intellectual classicism, which would also become a feature of John Paul II's talent for ecumenical communication and his theological vision of the Church. In the transition from youthful sonnets to hymns, we can notice not only the stylistic development and depth of the message, but, above all, a move towards conscious religiousness, contemplation, towards the priesthood, a move which was born in the very dramatic time of the Second World War. As a priest, on the other hand, his poetic choices were marked by a desire to understand the condition of man and to see God's love for His creation. This contemplation becomes increasingly more like a prayer, and dialogue is born out of prayer. Karol Wojtyła's literary work appears as a story about his own inner experience and reveals a spiritual anthropology.

Keywords: Karol Wojtyła; John Paul II; literary work; poetry; criticism and interpretation 